\title{
A random gradient based Harmony search algorithm for long- term hydropower scheduling in the lower of Jinsha River Basin
}

\author{
Yu Feng ${ }^{1,2,3}$, Jijun $\mathrm{Xu}^{1,2, *}$, Jin Chen ${ }^{1,2}$, Yongqiang Wang ${ }^{1,2}$, Xiaofeng Hong ${ }^{1,2}$ \\ ${ }^{1}$ Hubei Provincial Key Laboratory of Basin Water Resources and Ecological Environment, Changjiang River Scientific Research Institute, Wuhan, China, \\ 430010, China \\ ${ }^{2}$ Changjiang River Scientific Research Institute of Changjiang Water Resources Commission, Wuhan 430010, China \\ ${ }^{3}$ School of Hydropower and Information Engineering, Huazhong University of Science and Technology, Wuhan, Hubei 430074, China
}

\begin{abstract}
The long-term hydropower generation scheduling (LHGS) is one of the most important problems which aim to obtain a suitable scheme to maximize electrical benefits. In this paper, a gradient based Harmony search algorithm (GHS) is employed on the optimization of the cascade hydropower stations in the Jinsha River. Firstly, the original Harmony search algorithm (HS) is firstly improved by a dynamically adjustment strategy and global pitch adjustment strategy. Then random gradient strategy is adopted to improve the search speed of HS. In case study, the proposed GHS is used to solve the LHGS problem in the Jinsha River. Results show that GHS is effectiveness when dealing with LHGS problems.
\end{abstract}

\section{Introduction}

Hydropower is an important renewable energy source and plays an important role in the power grid. Statistics show that the total installed capacity of hydropower in China has reached 31.954 million kilowatts in 2015. As an important hydropower base, some hydropower stations in the lower reaches of the Jinsha River have been put into operation. It is of great importance to the long-term hydropower generation scheduling (LHGS) of the four hydropower stations in the Jinsha River.

The goal of long-term hydropower generation scheduling (LHGS) is to determine the process of water release in order to maximum the power generation. Over the past decade, there have been many researches on the dispatching of cascade hydropower stations, and indicating that LHGS is a non-linear problem with complicate constraints [1] which make LHGS difficult to solve. Classic methods such as dynamic programming (DP) [2] and progressive optimality algorithm (POA) [3] have played an important role in solving LHGS, but they perform not well when deal with cascade hydropower stations [4]. Recent attention has focused on metaheuristic algorithms which have widely been applied to many areas [5]. Harmony Search (HS) is a phenomenonmimicking meta-heuristic proposed by Zong Woo Geem in 2001 [6]. It performed based on musical performances and is an effective way to solve the LHGS problem. Comparing with Genetic Algorithm (GA), HS searches faster and can overcome the drawback of GA's building block theory. Thus, HS has been applied to solve variety of problems.
Although HS has its advantages, it still has some shortcomings. When dealing with large-scaled cascade hydropower stations, HS performs local convergence which makes the results not satisfactory and the proper computational time cannot be guaranteed.

In order to overcome the shortcomings, in this paper, a random gradient based Harmony search algorithm (GHS) is proposed to solve the LHGS problem. Firstly, the original HS is improved by a dynamically adjustment strategy and global pitch adjustment strategy. However, when applying HS to LHGS, the results of HS are not satisfactory. Thus a random gradient strategy which improves the local search ability of HS is designed. This strategy improves the HS by adjusting the water level process with random disturbance. In case study, the proposed GHS is applied to the LHGS of the four hydropower stations in the Jinsha River. Comparison between different methods has been made. The results obtained by GHS are better than that obtained by CS, GSA and HS, indicating that GHS is effectiveness when dealing with LHGS problems.

\section{Problem Formulation}

\subsection{Objective function}

The main objective of LHGS is to maximize the electricity generation of the cascade hydropower stations:

$W=\max \sum_{t=1}^{T} \sum_{n=1}^{N} k_{i} Q_{i, t} H_{i, t} \Delta t$

* Corresponding author: Jinjun Xu, Email:xujj07@163.com 
where $W$ is the total electric energy production of the cascade hydropower stations, $k_{i}$ is the comprehensive benefit coefficient of hydropower station $i, Q_{i, t}$ is the generation flow and $H_{i, t}$ is the net water head. $\Delta t_{i}$ is the length of each time period.

\subsection{Constraints}

(1) Water balance constraint:

The storage capacity of the hydropower station $i$ at time $t$ and $t$ - 1 satisfy:

$$
\begin{gathered}
V_{i, t-1}+\left(I_{i, t} \cdot \Delta t\right)=V_{i, t}+\left(O_{i, t} \cdot \Delta t\right) \\
O_{i, t}=Q_{i, t}+S_{i, t}
\end{gathered}
$$

where $V_{i, t-1}$ and $V_{i, t}$ is the storage capacity of the hydropower station $i$ at time $t-1$ and time $t$ respectively. $I_{i, t}$ is the inflow and $O_{i, t}$ is the outflow. $\Delta t$ is the length of time period. $S_{i, t}$ is the spillage.

(2) Boundary constraints

The outflow, water level and power output are limited between there upper and lower boundaries:

$$
\begin{aligned}
& O_{i, t}^{\min } \leq O_{i, t} \leq O_{i, t}^{\max } \\
& Z_{i, t}^{\min } \leq Z_{i, t} \leq Z_{i, t}^{\max } \\
& P_{i, t}^{\min } \leq P_{i, t} \leq P_{i, t}^{\max }
\end{aligned}
$$

(3) Spillage constraint

$$
S_{i, t} \geq 0
$$

(4) Water level variation constraint

$$
\left|Z_{i, t}-Z_{i, t-1}\right| \leq Z_{i, t}^{\text {step }}
$$

\section{Methodology}

In this section, a random gradient strategy is proposed to improve the performance of Harmony search algorithm for solving LHGS problems.

\subsection{Improvement for Harmony search algorithm}

To improve the performance of HS, the useful information from the best individual is considered to improve the local search ability. Then the new individual is generated follows:

$$
x_{i}^{\prime}= \begin{cases}x_{i}+f w \cdot \operatorname{rand}(-1,1), & \operatorname{rand}(0,1)<\mathrm{p} \\ x_{i}+b w \cdot\left(x_{i}^{\text {best }}-x_{i}\right), & \text { otherwise }\end{cases}
$$

where $b w$ is a random number between 0 and $1, p$ is the crossover probability which is dynamically adjusted follows:

$$
p=p_{s}+\left(p_{e}-p_{s}\right) \times \frac{C I}{N I}(10)
$$

where $C I$ is the current iteration, $N I$ is the maximum iteration, $p_{s}$ and $p_{e}$ are start and end crossover probability respectively. Under this evolutionary mechanism, the new individual has higher probability to be affected by the best individual when $p$ is small. After a number of numerical experiments, we set the $p_{s}=0.9, p_{e}=0.4$, meaning that the algorithm will have better local search ability in the later stage.

\subsection{Random gradient strategy}

When applying HS to the LHGS problem, the new individuals of the next generation are randomly generated following the characteristics of the algorithm itself. After numbers of experiments, it is found that the convergence speed is not very satisfactory when solving LHGS problem. Thus a random gradient strategy is designed to improve the convergence speed of HS for LHGS.

Following the random gradient strategy, the individual generated by the pitch adjustment will be adjusted by a random disturbance $\Delta l$. If the new individual after adjustment is better than before, repeat the adjustment again until the new individual is not better than the old one. The flowchart of the random gradient strategy is shown in Figure 1.

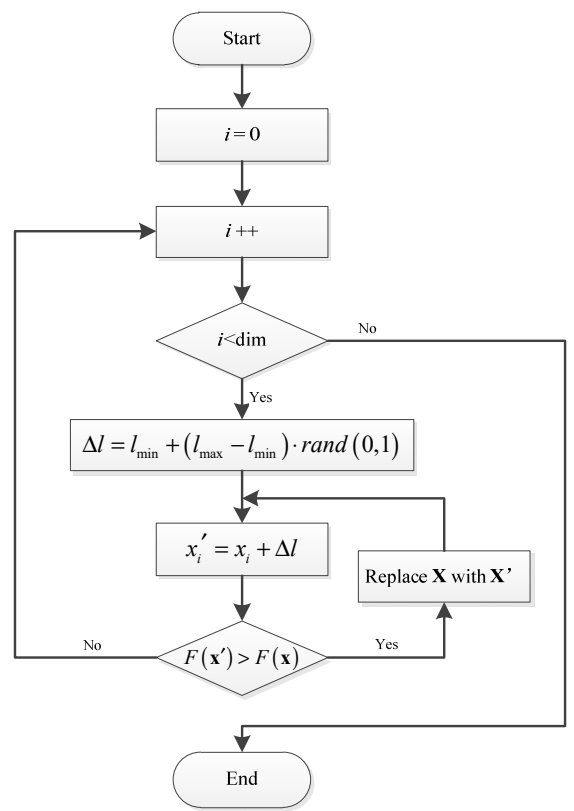

Figure 1. Random gradient strategy

\subsection{Random gradient based Harmony search algorithm}

According to the modification above, the steps of the algorithm are shown as follows:

Step 1: Randomly generate initial population and evaluate fitness of the individuals.

Step 2: Generate new solutions using memory consideration and pitch adjustment methods.

Step 3: Adjust the new solution following random gradient strategy.

Step 4: Compare the new individual with the worst individual in the population and update the population.

Step 5: Repeat Steps 2 to 4 until the stop criteria is reached. 


\section{Case study}

In this section, the proposed GHS is applied to the LHGS problem of four hydropower stations in the Jinsha River, including Wudongde, Baihetan, Xiluodu and Xiangjiaba. Topology of the four hydropower stations is shown in Figure 2. Three typical historical observed streamflow shown in Figure 3 are used as the input of LHGS model. Each year is divided into 36 time intervals. The parameter of GHS are $h m c r=0.9, p a r=0.3, f w=$ 0.005 , and the maximum number of iterations is set to 300. Scheduling results obtained by GHS are compared with HS, CS [7] and GSA [8]. Details can be seen in Table 1.

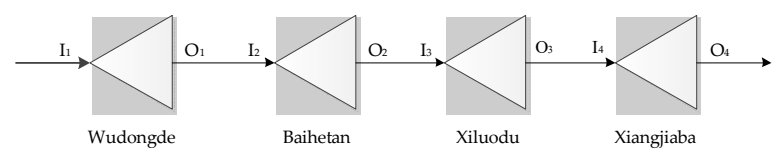

Figure 2. Topology of the cascade hydropower stations

Table 1 shows that GHS is superior to the other three methods. The average power generation obtained by GHS are 222.0, 207.8 and 199.2 billion $\mathrm{kWh}$ in three typical years. Compared with CS, GSA and HS, the average increases are $0.7 \%, 4.6 \%$ and $2.6 \%$, respectively. Moreover, the standard deviations obtained by GHS are also smaller than that obtained by the other three methods, thus indicating GHS is more stable.

Figure 4 shows the comparison of convergence process in the case of normal year as an example. It can be seen that GHS has the fastest convergence speed, CS and HS have not converged yet, while GSA has fallen into a local convergence.

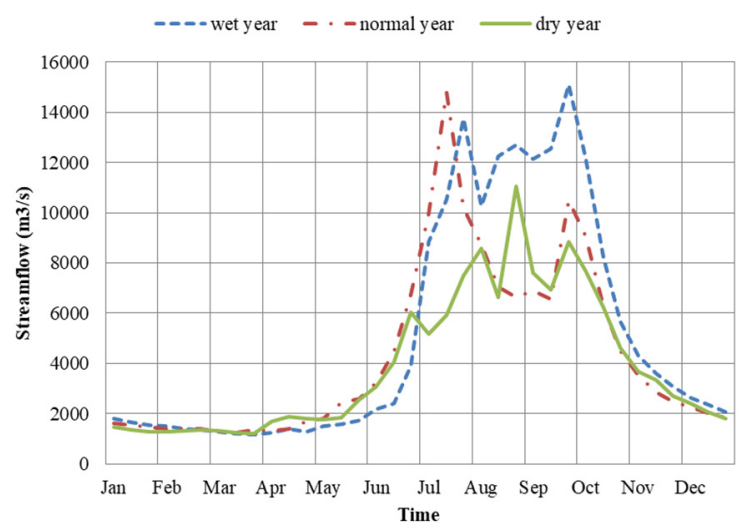

Figure 3. Streamflow in typical years

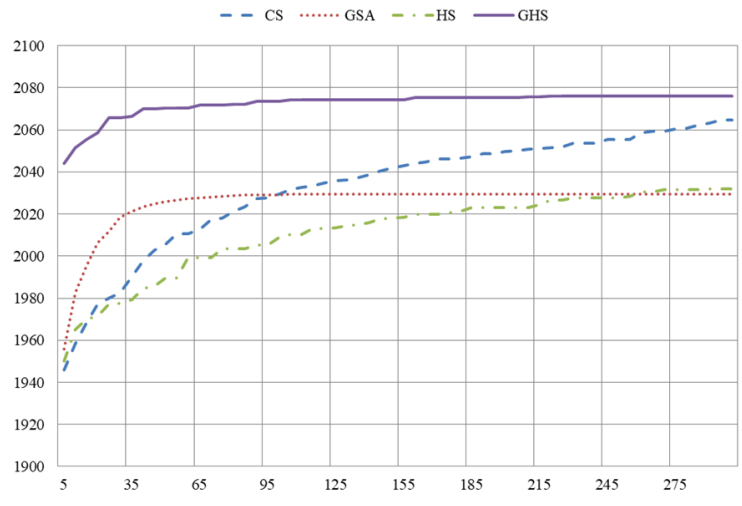

Figure 4. Streamflow in typical years

Figure 5 shows the optimal results of the cascade hydropower stations in the normal year as an example. As shown in Figure 5, water levels dropped below the flood limit water level during the flooding season. In the non-flooding season, the water levels keep near the normal level from October to February. It can also be seen from the figure that the change of water level obtained by GHS is small, indicating that the GHS perform well in dealing with LHGS.

Table 1 Comparison of power generation $\left(10^{8} \mathrm{kWh}\right)$ by different methods

\begin{tabular}{ccccccc}
\hline \multirow{2}{*}{ Alghorithm } & \multicolumn{2}{c}{ Wet year } & \multicolumn{2}{c}{ Normal year } & \multicolumn{2}{c}{ Dry year } \\
\cline { 2 - 6 } & Mean & Std. & Mean & Std. & Mean & Std. \\
\hline CS & 2211 & 11.60 & 2064 & 6.70 & 1973 & 6.95 \\
GSA & 2133 & 12.59 & 1987 & 6.40 & 1890 & 5.69 \\
HS & 2164 & 5.13 & 2025 & 3.17 & 1939 & 4.87 \\
GHS & 2220 & 5.72 & 2078 & 0.35 & 1992 & 1.86 \\
\hline
\end{tabular}




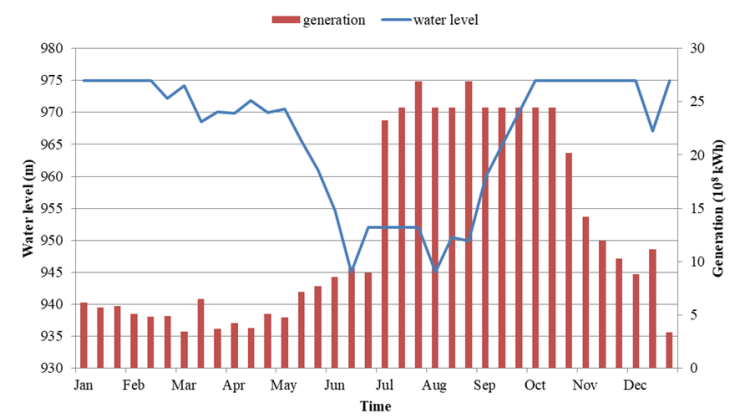

(1) Wudongde

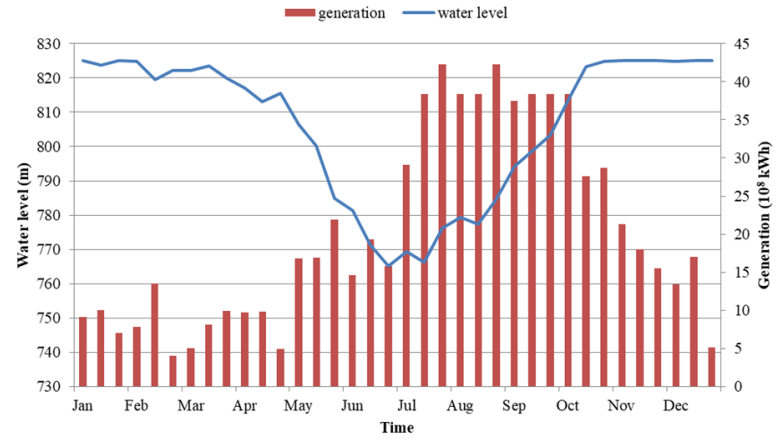

(2) Baihetan

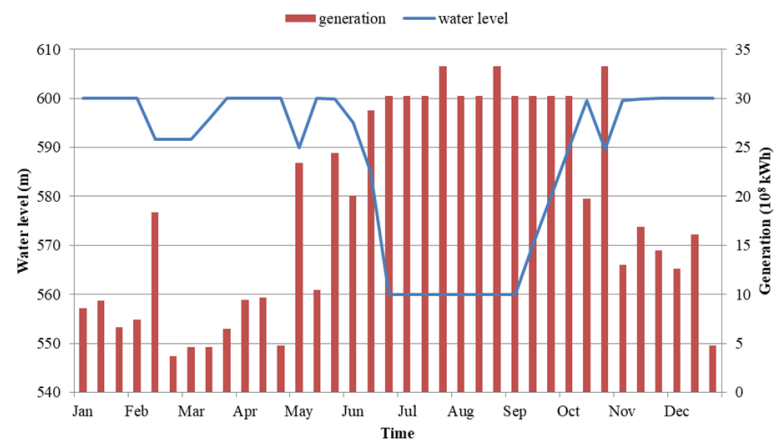

(3) Xiluodu

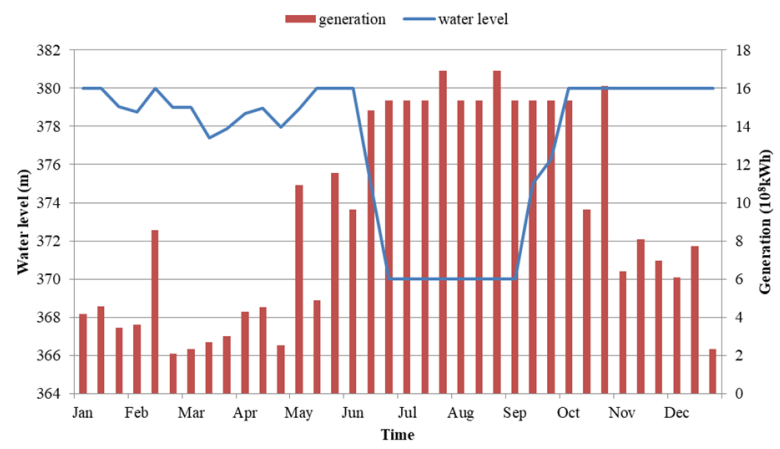

(4) Xiangjiaba

Figure 5. The optimal results obtained by GHS.

\section{Conclusion}

In this paper, a random gradient based harmony search algorithm is proposed to deal with the long-term hydropower scheduling problem in Jinsha River. The proposed method can effectively obtain an optimal scheduling scheme which meets the requirement for hydropower generation. Using the random gradient strategy, the harmony search algorithm can converge faster when dealing with LHGS. In case study, the comparisons among different methods show that the results obtained by GHS are more reliable than other three methods. Overall, GHS is an effective way to solve LHGS. Further research is underway to consider more objective such as guaranteed output. 


\section{Acknowledgements}

This work is funded by the National Key R\&D Program of China(2017YFC0403600, 2017YFC0403606), the National Natural Science Foundation of China (No. 51779013, 51509009), National Public Research Institutes for Basic R\&D Operating Expenses Special Project (No.CKSF2017008, CKSF2016050), Water Conservancy Science and Technology Innovation project of GuangDong Province(2017-03). Special thanks are given to the anonymous reviewers and editors for their constructive comments.

\section{References}

1. Liao X, Zhou J, Zhang $\mathrm{R}$, et al. An adaptive artificial bee colony algorithm for long-term economic dispatch in cascaded hydropower systems. International Journal of Electrical Power \& Energy Systems, 2012, 43(1): 1340-5.

2. Soares S, Ohishi T, Cicogna $\mathrm{M}$, et al. Dynamic dispatch of hydro generating units; proceedings of the Power Tech Conference Proceedings, 2003 IEEE Bologna, F, 2003 [C].

3. Nanda J, Bijwe P R. Optimal Hydrothermal Scheduling with Cascaded Plants Using Progressive Optimality Algorithm. IEEE Transactions on Power Apparatus \& Systems, 1981, PAS-100(4): 2093-9.

4. Wang C, Zhou J, Peng L, et al. Long-term scheduling of large cascade hydropower stations in Jinsha River, China. Energy Conversion \& Management, 2015, 90(2015): 476-87.

5. Beck A T, Gomes W J D S. A comparison of deterministic, reliability-based and risk-based structural optimization under uncertainty. Probabilistic Engineering Mechanics, 2012, 28(4): 18-29.

6. Zong W G, Kim J H, Loganathan G V. A New Heuristic Optimization Algorithm: Harmony Search. Simulation, 2001, 2(2): 60-8.

7. Yang X S, Deb S. Cuckoo Search via Levy Flights. Mathematics, 2010: 210 - 4.

8. Rashedi E, Nezamabadi-Pour H, Saryazdi S. GSA: A Gravitational Search Algorithm. Intelligent Information Management, 2012, 4(6): 390-5. 